\title{
Survey study on the practice patterns in the endoscopic management of malignant distal biliary obstruction
}

\section{(ㄷ)(1) $\odot$}

Authors

Dennis Yang1, Yaseen B. Perbtani ${ }^{1}$, Qi An ${ }^{2}$, Mitali Agarwal ${ }^{3}$, Michael Riverso', Joydeep Chakraborty ${ }^{3}$, Tony S. Brar ${ }^{3}$, Donevan Westerveld ${ }^{3}$, Han Zhang ${ }^{3}$, Shailendra S. Chauhan ${ }^{4}$, Christopher E. Forsmark ${ }^{1}$, Peter V. Draganov ${ }^{1}$

Institutions

1 Division of Gastroenterology and Hepatology, University of Florida, Gainesville, FL, USA

2 Department of Health Outcomes and Policy, University of Florida, Gainesville, FL, USA

3 Department of Medicine, University of Florida, Gainesville, FL, USA

4 CHS Digestive Health, Carolinas Medical Center, Charlotte, NC, USA

submitted 3.1.2017

accepted after revision 23.3.2017

Bibliography

DOI https://doi.org/10.1055/s-0043-111592 |

Endoscopy International Open 2017; 05: E754-E762

(c) Georg Thieme Verlag KG Stuttgart · New York

ISSN 2364-3722

Corresponding author

Dennis Yang, MD, Division of Gastroenterology, University of Florida, 1329 SW 16th Street, Suite 5251, Gainesville, FL 32608, USA

Fax: +1-352-627-9002

Dennis.Yang@medicine.ufl.edu

\section{ABSTRACT}

Background and study aim Endoscopic biliary drainage for malignant distal biliary obstruction (MDBO) is a common practice. Controversy persists with regard to its role in resectable $\mathrm{MDBO}$, the optimal technical method and type of stent. The aim of this study was to evaluate practice patterns in the treatment of MDBO among endoscopists with varying levels of experience and practice backgrounds. Methods Electronic survey distributed to members of the American Society for Gastrointestinal Endoscopy (ASGE). The main outcome measures included practice setting (academic vs. community), volume of endoscopic retrograde cholangiopancreatographies (ERCPs), reasons for endoscopic drainage in MDBO, and technical approach.

Results A total of 335 subjects (54\% community-based endoscopists) completed the survey. Most academic physicians (69\%) reported performing $\geq 150$ ERCPs annually compared to $18.8 \%$ of community physicians $(P<0.001)$. In aggregate, $13.1 \%$ of respondents performed ERCP in resectable MDBO because of surgeon preference or as the standard of care at their institution. The use of metal vs. plastic stents in MDBO varied based on practice setting. Routine sphincterotomy for MDBO was more common among community $(78 \%)$ vs academic endoscopists $(61.1 \%)(P<0.001)$. Over half ( $58 \%$ ) of the subjects avoided covering the cystic duct take-off during stenting MDBO if there was a gallbladder in situ.

Conclusion There is significant variability in practice patterns for the treatment of MDBO. In spite of the recent ASGE guideline recommendations, some patients with resectable MDBO still undergo preoperative ERCP. Current clinical practices are not clearly supported by available data and underscore the need to increase adherence to gastrointestinal societal recommendations and an evidence-based approach to standardized patient care.

\section{Introduction}

Malignant biliary obstruction is a complex clinical condition commonly encountered in practice. Bile duct luminal narrowing can occur as a consequence of intrinsic tumor growth (i.e. cholangiocarcinoma) or from external compression from adjacent neoplasms and/or lymphadenopathy. Overall, pancreatic neoplasms account for most cases of malignant distal biliary obstruction (MDBO) [1].
Endoscopic stenting is commonly regarded as the mainstay management strategy of MDBO. The therapeutic goal is to achieve biliary decompression to treat and prevent adverse events associated with jaundice. While endoscopic stenting for MDBO is commonly performed, its role in patients with resectable disease is questionable [2,3]. Indeed, most clinical trials have failed to demonstrate any significant benefit of routine preoperative biliary drainage in patients with resectable MDBO and have suggested increased morbidity associated with this 
practice [4-9]. In light of the current evidence, the Standards of Practice Committee of the American Society for Gastrointestinal Endoscopy (ASGE) recently updated their guidelines recommending against routine preoperative endoscopic retrograde cholangiopancreatography (ERCP) in patients with asymptomatic obstructive jaundice [10]. Nonetheless, there remains wide variability on other technical aspects of preoperative ERCP, including the choice of biliary endoprosthesis or the need for routine biliary sphincterotomy. The aim of this study was to evaluate preferences and practice patterns in the management of MDBO among endoscopists with varying levels of experience and from different practice backgrounds.

\section{Methods}

This study was approved by the University of Florida Institutional Review Board.

\section{Study population and study design}

This study was designed as an electronic survey. Email addresses from the membership directory of the American Society for Gastrointestinal Endoscopy (ASGE) were used to target the study participants. The ASGE members were then contacted through email via the University of Florida Research Electronic Data Capture (REDCapTM) web-based software and invited to complete the voluntary anonymous electronic survey questionnaire. The survey tool was designed to be completed in approximately 5 minutes. Reminder emails requesting participation were automatically sent every 2 weeks to subjects who had not completed the survey (a total of no more than three reminder emails were sent over the course of 6 weeks). An incentive program for participation consisted of distributing a personal smart tablet computer to each 100th subject who completed the online survey tool. The survey was launched between February 2016 and March 2016 and was closed after 8 weeks.

\section{Electronic survey instrument}

The 15-item survey questionnaire included items related to the subjects' advanced endoscopic training, practice setting, volume of endoscopic retrograde cholangiopancreatographies (ERCPs) performed annually and specifically for MDBO, commonly encountered reasons for ERCP in patients with MDBO in their practice, their preferences in the type of endoluminal stent (i.e. plastic, metal) and techniques (i.e. biliary sphincterotomy prior to stent insertion, position of stent in the setting of gallbladder in situ) for the treatment of MDBO, and their approach after initial failed ERCP. A copy of the electronic survey questionnaire can be viewed in Supplementary File 1.

\section{Statistical analysis}

All responses were included for analysis when possible. Blank responses to individual questions were excluded from the analysis of that question. All responses to surveys were summarized descriptively. Two-sided chi-square analysis was used to test for differences for all categorical variables whereas the two-sided Wilcoxon rank test was used to assess for differences for all continuous characteristics. A $P$ value of $<0.05$ was considered statistically significant. All statistical analyses were performed with SAS 9.4 software (Cary, North Carolina, United States).

\section{Results}

\section{Baseline characteristics}

The electronic survey tool was sent to 4874 email addresses through the ASGE membership directory of which 335 subjects (6.9\%) responded. Approximately one third of all participants (112/335; 33.4\%) completed a 4th year advanced endoscopy fellowship. More than half $(181 / 335 ; 54 \%)$ of the respondents identified themselves as community-based endoscopists while $43 \%$ (144/335) were academic/university-based. Ten participants ( $3 \%$ ) practiced in the Veterans Affairs (VA) hospital setting. Overall, $70.1 \%$ (235/335) of respondents reported performing more than 75 ERCPs yearly. There was a statistically significant difference in the volume of ERCPs per year between academic- and community-based practitioners ( $\triangleright$ Table 1 ). Most academic endoscopists $(98 / 144 ; 68.1 \%)$ reported performing more than 150 ERCPs yearly compared to only $18.8 \%$ $(36 / 191)$ of those from a community-based practice $(P<$ 0.001). High-volume ERCPs for MDBO (defined as more than 20 annually) were also more commonly seen among academic endoscopists $(110 / 144 ; 76.4 \%)$ compared to those from the community $(62 / 191 ; 32.5 \%)(P<0.001)$.

\section{Common indications for ERCP in distal MDBO}

Overall, $42.7 \%$ (143/335) of respondents reported that surgeons routinely referred patients with resectable MDBO for preoperative ERCP. This practice was significantly more frequent in the academic $(86 / 144 ; 59.7 \%)$ than in the community (57/191; 29.8\%) setting $(P<0.001)$.

- Fig. 1 depicts reasons for ERCP in patients with resectable MDBO based on practice environment. Approximately half (68) $144 ; 47.2 \%)$ of academic endoscopists cited the need for neoadjuvant therapy prior to surgery as the most common indication for ERCP compared to only $16.8 \%$ (32/191) in the community $(P<0.001)$. Conversely, ascending cholangitis was ranked as the most common indication for ERCP among community-based practitioners $(68 / 191 ; 35.6 \%)$. The frequency of ERCPs performed for other reasons was similar between the two groups ( $\triangleright$ Fig. 1 ). In aggregate, $44 / 335$ (13.1\%) of all respondents indicated that ERCP was performed because the "surgeon prefers lower total bilirubin prior to surgery" or it is "standard of care" at their respective institution.

\section{Types of biliary stents and ERCP techniques in MDBO}

Participants were asked about the most commonly used type of stent in their practice for the management of both resectable and unresectable MDBO. More than half of all respondents (195/335; 58.2\%) preferred plastic over metal stents for resectable lesions whereas metal stents were almost exclusively chosen for unresectable disease $(327 / 335 ; 97.6 \%)$. There were significant differences in stent choice based on practice setting ( Table 2). For resectable lesions, 62.5\% (90/144) of academic-based endoscopists preferred metal over plastic biliary stents whereas the plastic type was more commonly used in 
- Table 1 Annual ERCP volume among respondents based on type of practice environment.

\begin{tabular}{|c|c|c|c|}
\hline & Academic/university-based practice $(n=144)$ & Community-based practice $(n=191)$ & $P$ value \\
\hline \multicolumn{4}{|c|}{ Annual ERCP volume } \\
\hline $25-75, \mathrm{n}(\%)$ & $16(11.1)$ & $84(44)$ & \\
\hline $75-150, n(\%)$ & $29(20.1)$ & $71(37.2)$ & $<0.001$ \\
\hline$>150, \mathrm{n}(\%)$ & $98(681)$ & $36(18.8)$ & \\
\hline \multicolumn{4}{|c|}{ Annual ERCP volume for MDBO } \\
\hline$<5, \mathrm{n}(\%)$ & $4(2.8)$ & $38(19.9)$ & \\
\hline $6-10, n(\%)$ & $6(4.2)$ & $46(24.1)$ & $<0.001$ \\
\hline $11-20, \mathrm{n}(\%)$ & $23(16)$ & $45(23.6)$ & \\
\hline$>20, \mathrm{n}(\%)$ & $110(76.4)$ & $62(32.5)$ & \\
\hline
\end{tabular}

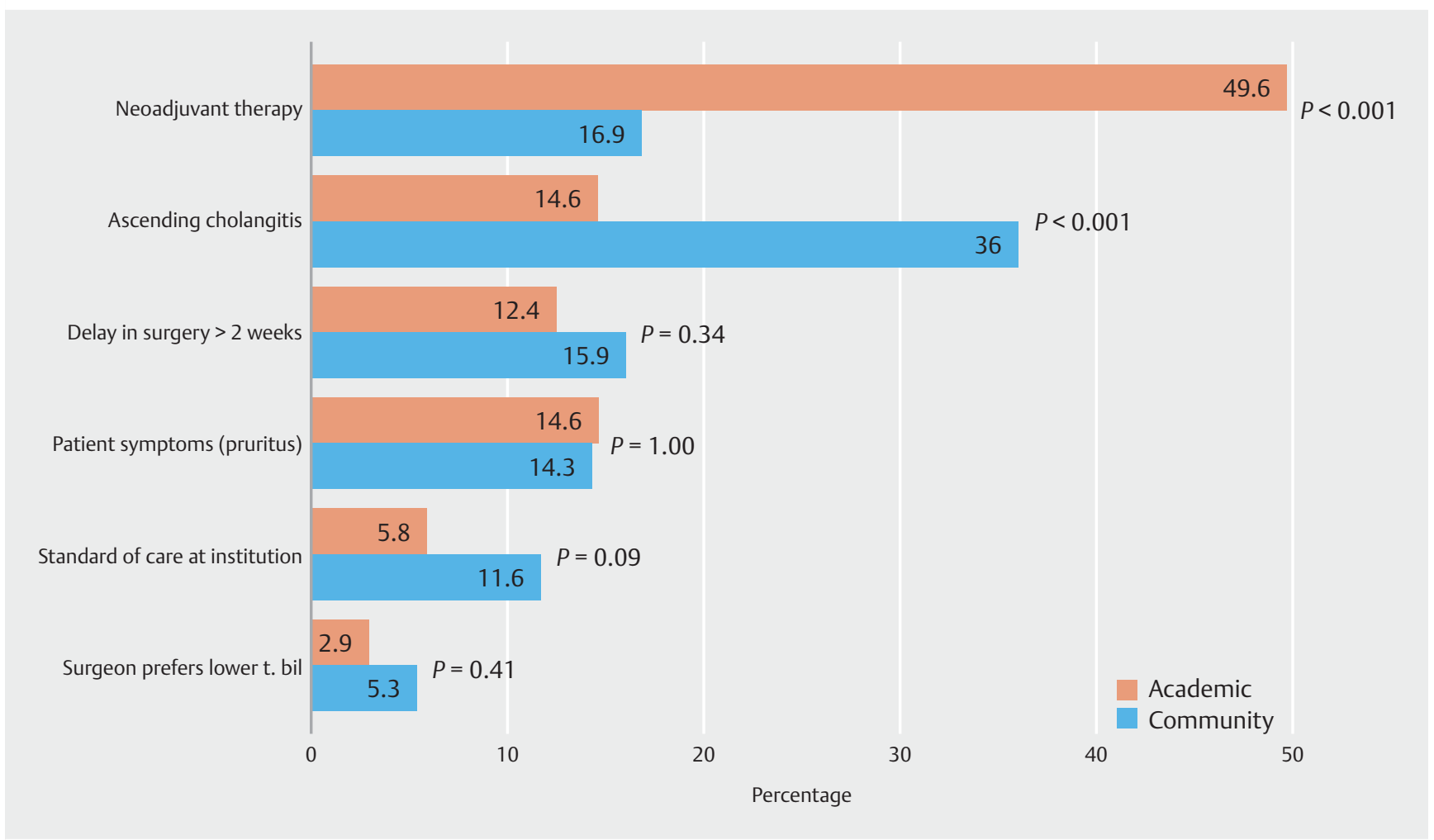

- Fig. 1 Common reasons for endoscopic biliary drainage in resectable MDBO.

the community $(143 / 191 ; 74.9 \%)(P<0.001)$. For unresectable MDBO, uncovered metal stents were preferred by academicbased practitioners $(85 / 144 ; 59 \%)$ whereas community-based endoscopist preferred fully-covered $(90 / 191 ; 47.1 \%)$ followed by partially-covered metal stents $(58 / 191 ; 30.4 \%)(P<0.001)$.

Slightly more than half of all participants responded that the type of stent used would not depend on whether there was a gallbladder in situ (189/335; 56.4\%), yet most agreed that they would avoid covering the cystic duct take-off when placing a covered biliary stent (194/335; 57.9\%). Most subjects re- ported frequently performing routine biliary sphincterotomy prior to stent insertion for MDBO (236/335; 70.4\%), with this practice being significantly more common in the community vs the academic setting (77.5\% vs $61.1 \%)(P=0.001)$.

The overall approach after an initial failed attempt at ERCP for resectable MDBO was similar between endoscopists from the academic and community setting ( $\triangleright$ Table 3 ). Repeating the ERCP by themselves or by another endoscopy center was the most common approach cited by academic (71/144; $49.3 \%)$ and community $(77 / 191 ; 40.3 \%)$ endoscopists $(P=0.12)$. On 
Table 2 Preferences on the type of stents for resectable and unresectable MDBO based on the type of practice environment.

\begin{tabular}{|l|l|l|l|l|l|}
\hline Type of stent & \multicolumn{2}{|c|}{ Resectable MDBO } & P value & \multicolumn{2}{c|}{ Unresectable MDBO } \\
\hline & Academic & Community & & Academic & Community \\
\hline Plastic, n (\%) & $52(36.6)$ & $143(75.7)$ & $<0.001$ & $6(4.2)$ & $2(1)$ \\
\hline PC-SEMS, n (\%) & $4(2.8)$ & $6(3.2)$ & NS & $15(10.4)$ & $58(30.4)$ \\
\hline FC-SEMS, n (\%) & $58(40.8)$ & $36(19)$ & $<0.001$ & $38(26.4)$ & $90(47.1)$ \\
\hline U-SEMS, $n$ (\%) & $28(19.7)$ & $4(2.1)$ & $<0.001$ & $85(59)$ & $41(21.5)$ \\
\hline
\end{tabular}

MDBO, malignant distal biliary obstruction; PC-SEMS, partially covered self-expandable metal stents; FC, fully-covered; U, uncovered; NS, not significant.

\begin{tabular}{|l|l|l|l|l|l|l|}
\hline \begin{tabular}{l} 
Table 3 Treatment approach after initial failed ERCP for MDBO. \\
\hline Treatment approach
\end{tabular} & Resectable MDBO & P value & Unresectable MDBO & Community \\
\hline & Academic & Community & & Academic & N value \\
\hline Repeat ERCP, $\mathrm{n}(\%)$ & $71(49.3)$ & $77(40.3)$ & NS & $54(37.5)$ & $74(38.7)$ \\
\hline Refer for PTC, $\mathrm{n}(\%)$ & $28(19.4)$ & $42(22)$ & NS & $39(27.1)$ & $85(44.5)$ \\
\hline Directly to surgery, $\mathrm{n}(\%)$ & $24(16.7)$ & $56(29.3)$ & 0.009 & $2(1.4)$ & 0 & 0.001 \\
\hline Refer for EUS-guided biliary drainage, $\mathrm{n}(\%)$ & $21(14.6)$ & $16(8.4)$ & NS & $49(34)$ & $32(16.8)$ \\
\hline
\end{tabular}

ERCP, endoscopic retrograde cholangiopancreatography; MDBO, malignant distal biliary obstruction; PTC, percutaneous transhepatic cholangiography; EUS, endoscopic ultrasound; NS, not significant.

the other hand, direct referral to surgery following initial failed ERCP attempt was more common among community $(56 / 191 ; 29.3 \%)$ vs academic $(24 / 144 ; 16.7 \%)$ endoscopists $(P=0.009)$. There were some differences in the management approach to unresectable MDBO based on practice environment ( $\vee$ Table 3 ). Percutaneous transhepatic cholangiography (PTC) was more commonly cited as the next step after failed ERCP for unresectable disease by community-based practitioners $(85 / 191 ; 44.5 \%)$ when compared to those from an academic setting $(39 / 191 ; 20.4 \%)(P<0.001)$. Conversely, EUSguided biliary drainage for unresectable MDBO was almost twice more commonly chosen as the next step following failed ERCP by academic $(49 / 144 ; 34 \%)$ vs. community-based ( $32 /$ $191 ; 16.8 \%)$ endoscopists $(P<0.001)$.

\section{Discussion}

Endoscopic stenting is frequently performed for the management of malignant distal biliary obstruction (MDBO) with the goal of achieving biliary decompression. In spite of this common practice, considerable controversy persists over indications for preoperative ERCP in resectable disease, the optimal type of biliary stent, the need for biliary sphincterotomy, and the approach after an initial failed ERCP. This survey study demonstrated significant variability in the practice patterns for the treatment of MDBO among endoscopists of varying levels of experience and practice environments.

The role of endoscopic biliary drainage in patients with resectable MDBO is questionable. In a landmark multicenter pro- spective study by Van der Gaag et al., the rates of serious adverse events was significantly higher in patients randomized to preoperative biliary drainage vs early surgery ( $74 \%$ vs $39 \%$; $95 \%$ Cl: $0.41-0.71 ; P<0.001)[4]$. While this initial study has been scrutinized based on its unusually high adverse event rates and the use of plastic instead of metal biliary stents, subsequent studies have not shown an advantage for preoperative biliary drainage [5-9]. In a systematic review including six randomized clinical trials $(n=520)$, Fang and colleagues also reported a higher incidence of adverse events among patients in the preoperative biliary drainage group vs direct surgery group $73.5 \%$ vs $37.4 \%$; $P<0.001$ ) [5]. Based on the current evidence, the ASGE recommends against routine preoperative ERCP apart from those requiring neoadjuvant therapy, presenting with acute cholangitis, or in whom surgery will be delayed [10-12]. In our study, approximately $43 \%$ (143/335) of all respondents indicated that surgeons frequently referred patients for preoperative endoscopic biliary drainage in patients with resectable MDBO. While the vast majority of these procedures were performed in select patients (i.e. need for neoadjuvant therapy, patient's symptoms due to jaundice, delayed surgery), $13.1 \%$ of respondents (44/335) indicated that the reason for the ERCP was either "surgeon prefers lower total bilirubin prior to surgery" or "standard of care at their institution". These findings indicate that some patients with resectable MDBO may still be undergoing routine preoperative biliary drainage and emphasize the importance of implementing evidence-based practice to limit an indiscriminate approach to preoperative endoscopic drainage in resectable MDBO, given its association with 
increased morbidity, additional costs, and delay in surgery [13, 14].

There was significant variability in the type of endobiliary stent used for the management of MDBO among respondents. In general, there has been an increasing tendency for patients with both resectable and borderline resectable pancreatic cancer to undergo neoadjuvant therapy. Indeed, the need for neoadjuvant therapy was the most commonly cited reason for preoperative drainage by academic-based endoscopists (49.6\% vs $16.9 \%$ in the community setting; $P<0.001)$. The difference in the proportion of patients undergoing neoadjuvant therapy as reported in this survey may potentially explain why metal biliary stents were more commonly preferred by academic (62.5\%) vs community-based $(24.3 \%)$ practitioners $(P<0.001)$. Self-expanding metal stents (SEMS) may be associated with lower stent occlusion rates when compared to plastic stents given their larger caliber and may be preferable in the setting of neoadjuvant therapy $[15,16]$. Not surprisingly, nearly all respondents in this study preferred SEMS (94.8\%) over plastic stents (5.2\%) for unresectable MDBO. Interestingly, more than half of academic-based endoscopists (59\%) preferred uncovered SEMS whereas most practitioners in the community opted for fully or partially covered SEMS (77.5\%) $(P<0.001)$. Traditionally, uncovered SEMS have been associated with a low migration rate but high rates of stent occlusion whereas covered SEMS may have lower rates of tissue ingrowth but an increased risk for migration. The current literature comparing these stents is conflicting $[17,18]$, which is reflected on the variable preferences for type of stents among respondents in this study.

This survey study assessed preferences in ERCP techniques during endobiliary stenting for MDBO. More than half of all respondents (57.9\%) reported that they would avoid covering the cystic duct take-off in the setting of a gallbladder in situ. These findings emphasize the prevalent concern among endoscopists for acute cholecystitis due to the occlusion of the cystic duct take-off with the biliary prosthesis. However, there is currently no evidence to strongly suggest an association between the type of biliary stent used and the risk of acute cholecystitis. In fact, tumor involvement of the cystic duct orifice has been the only factor positively correlated with the rate of acute cholecystitis in these patients [19-21]. In aggregate, most participants (70.4\%) indicated that they frequently perform biliary sphincterotomy prior to stent insertion, with a higher rate among community $(77.5 \%)$ vs academic $(61.1 \%)$ endoscopists $(P<$ $0.001)$. In theory, biliary sphincterotomy may reduce the compressive force exerted on the pancreatic duct orifice. However, the currently available literature does not clearly support a protective effect against post-ERCP pancreatitis; especially in those with MDBO from pancreatic cancer [22-24]. On the other hand, biliary sphincterotomy may be necessary in certain circumstances in order to facilitate stent insertion or advancement of endoscopic devices (i.e. cytology brush, biopsy forceps). Given the low yet serious risks associated with biliary sphincterotomy, this should not be indiscriminately performed but rather determined on a case-by-case basis. Further prospective randomized studies designed to address the association between acute cholecystitis and pancreatitis during ERCP are desirable, yet challenging, given the prohibitive number of subjects that may be required given the low event rate of these adverse events.

The study has several strengths. Using an electronic survey tool, we were able to assess practice patterns on the endoscopic management of MDBO in light of the recent ASGE recommendations [10]. The results of this study provide further insight on the variability in practice patterns among endoscopists and the need to implement strategies to standardize the approach to the management of MDBO. We also acknowledge the limitations of this study. The survey tool was sent to all ASGE members with an active email account. This method most certainly did not capture every ERCP practitioner eligible for participation in the study, thereby introducing selection bias in our cohort. Furthermore, the survey was not sent exclusively to ASGE members who perform ERCP. Thus, it is not possible to ascertain the precise response rate for this study, although we theorize that this must have been higher than $6.9 \%$ as not all 4874 active email recipients perform this procedure. Nonetheless, we recognize that the results from this survey must be interpreted with caution and may not be representative given the low response rate. Lastly, this study is subject to the limitations associated with survey questionnaires, primarily the potential for recall and reporting bias.

In summary, this study found that there is significant variability in the volume of ERCPs and practice patterns for the treatment of MDBO among endoscopists from different practice environments. In spite of the recent ASGE guideline recommendations, our survey results indicate that some patients with resectable MDBO are still undergoing routine preoperative endoscopic biliary drainage. Routine biliary sphincterotomy for MDBO cannot be advocated as standard of practice but the decision to perform sphincterotomy should be individualized. Our study highlights the need to institute interventions to increase the adherence to ASGE guideline recommendations and an evidence-based approach in an effort to reduce the current variability in the treatment of MDBO.

\section{Competing interests}

P.V. Draganov is a consultant for Boston Scientific, Cook Medical, Olympus America, and Conmed; D. Yang is a consultant for US Endoscopy. All other authors disclosed no financial relationships relevant to this publication.

\section{References}

[1] Roque J, Ho SH, Goh KL. Preoperative drainage for malignant biliary strictures: is it time for self-expanding metallic stents? Clin Endosc 2015; 48: 8-14

[2] Pu LZ, Singh R, Loong CK et al. Malignant biliary obstruction: evidence for best practice. Gastroenterol Res Pract 2016; 2016: 3296801

[3] Almadi MA, Barkun JS, Barkun AN. Stenting in malignant biliary obstruction. Gastrointest Endosc Clin N Am 2015; 25: 691 - 711 
[4] Van der Gaag NA, Rauws EA, van Eijck CH et al. Preoperative biliary drainage for cancer of the head of the pancreas. NEJM 2010; 362: $129-137$

[5] Fang Y, Gurusamy KS, Wang Q et al. Meta-analysis of randomized clinical trials on safety and efficacy of biliary drainage before surgery for obstructive jaundice. Br J Surg 2013; 100: 1589-1596

[6] Velanovich V, Kheibek T, Khan M. Relationship of postoperative complications from preoperative biliary stents after pancreaticoduodenectomy. A new cohort analysis and meta-analysis of modern studies. JOP 2009; 10: $24-29$

[7] Hong SK, Jang JY, Kang MJ et al. Comparison of clinical outcome and cost-effectiveness after various preoperative biliary drainage methods in periampullary cancer with obstructive jaundice. J Korean Med Sci 2012; 27: $356-362$

[8] Qiu YD, Bai JL, Xu FG et al. Effect of preoperative biliary drainage on malignant obstructive jaundice: a meta-analysis. World J Gastroenterol 2011; 17: $391-396$

[9] Sewnath ME, Karsten TM, Prins MH et al. A meta-analysis on the efficacy of peroperative biliary drainage for tumors causing obstructive jaundice. Ann Surg 2002; 236: $17-27$

[10] ASGE Standards of Practice Committee. Eloubeidi MA, Decker GA et al. The role of endoscopy in the evaluation and management of patients with solid pancreatic neoplasia. Gastrointest Endosc 2016; 83: $17-28$

[11] Lai EC, Lau SH, Lau WY. The current status of preoperative biliary drainage for patients who receive pancreaticoduodenectomy for periampullary carcinoma: a comprehensive review. Surgeon 2014; 12: $290-296$

[12] Bonin EA, Baron TH. Preoperative biliary stents in pancreatic cancer J Hepatobiliary Pancreat Sci 2011; 18: 621-629

[13] Rustagi T, Jamidar PA. Endoscopic treatment of malignant biliary strictures. Curr Gastroenterol Rep 2015; $17: 3$

[14] Morris S, Gurusamy KS, Sheringham J et al. Cost-effectiveness of preoperative biliary drainage for obstructive jaundice in pancreatic and periampullary cancer. J Surg Res 2015; 193: $202-209$

[15] Siddiqui AA, Mehendiratta V, Loren D et al. Self-expanding metal stents (SEMS) for preoperative biliary decompression in patients with resectable and borderline-resectable pancreatic cancer: outcomes in 241 patients. Dig Dis Sci 2013; 58: 1744-1750

[16] Aadam AA, Evans DB, Khan A et al. Efficacy and safety of self-expandable metal stents for biliary decompression in patients receiving neoadjuvant therapy for pancreatic cancer: a prospective study. Gastrointest Endosc 2012; 76: 67 - 75

[17] Saleem A, Leggett CL, Murad MH et al. Meta-analysis of randomized trial comparing the patency of covered and uncovered self-expandable metal stents for palliation of distal malignant bile duct obstruction. Gastrointest Endosc 2011; 74: 321 - 327

[18] Almadi MA, Barkun AN, Martel M. No benefit of covered vs uncovered self-expandable metal stents in patients with malignant distal biliary obstruction: a meta-analysis. Clin Gastroenterol Hepatol 2013; 11 : $27-37$

[19] Lee JH, Kirshna SG, Singh A et al. Comparison of the utility of covered metal stents versus uncovered metal stents in the management of malignant biliary strictures in 749 patients. Gastrointest Endosc 2013; 78: $312-324$

[20] Shimizu S, Naitoh I, Nakazawa T et al. Predictive factors for pancreatitis and cholecystitis in endoscopic covered metal stenting for distal malignant biliary obstruction. J Gastroenterol Hepatol 2013; 28: 68 72

[21] Isayama H, Kawabe T, Nakai Y et al. Cholecystitis after metallic stent placement in patients with malignant distal biliary obstruction. Clin Gastroenterol Hepatol 2006; 4: 1148 - 1153

[22] Artifon EL, Sakai P, Ishioka S. Endoscopic sphincterotomy before deployment of covered metal stent is associated with greater complication rate: a prospective randomized controlled trial. J Clin Gastroenterol 2008; 42: 815-819

[23] Banerjee H, Hilden K, Baron TH et al. Endoscopic biliary sphincterotomy is not required for transpapillary SEMS placement for biliary obstruction. Dig Dis Sci 2011; 56: 591 - 595

[24] Hayashi T, Kawakami H, Osanai M et al. No benefit of endoscopic sphincterotomy before biliary placement of self-expandable metal stents for unresectable pancreatic cancer. Clin Gastroenterol Hepatol 2015; 13: $1151-1158$ 


\section{Supplement}

\section{Title of Project:}

Practice Patterns in the Endoscopic Management of Malignant Distal Biliary Obstruction: A Survey Analysis

\section{Principal Investigator:}

Dennis Yang, Assistant Professor of Medicine

Division of Gastroenterology, Hepatology and Nutrition

University of Florida

1. Purpose of the Study: The purpose of this study is to assess practice patterns for the management of malignant distal biliary obstruction among interventional endoscopists across the United States.

2. Procedures to be followed: You will be asked to answer 15 questions in this survey

3. Duration: It will take about 5 minutes to complete this survey

4. Research Benefits: There is no direct benefit to you for being in this study. There may be a benefit to others depending on the results of this study.

5. Research Risks: There is a risk that information received by these authorized persons or agencies could then be passed on to others beyond your authorization and not covered by HIPAA.

6. Statement of Confidentiality: Your participation in this research is confidential. The survey does not ask for any information that would identify who the responses belong to. In the event of any publication or presentation resulting from the research, no personally identifiable information will be shared because your name is in no way linked to your responses. All results will be analyzed and presented in aggregate form.

7. Right to Ask Questions: Please contact Dennis Yang at (352) 273-8212 or dennis.yang@medicine.ufl.edu with questions or concerns about this study.

8. Payment for participation: Each $200^{\text {th }}$ participant will be eligible for a smart tablet computer. To participate, please reply to the email invitation for this study once you have completed the survey. We will contact the subjects who will receive the tablet computer at the completion of the survey period (8 weeks after the initial invitation).

9. Privacy Authorization: UF and Shands will be allowed to collect, use and/or give out the information from this study, but only to the study investigators in this IRB approved study.

10. Voluntary Participation: Your decision to be in this research is voluntary. You can stop at any time. You do not have to answer any questions you do not want to answer. If you do not want to take part, you can call my office at any time to let me know $(352-273-8212)$ or you can tell me when I call you. If you choose not to take part, this will have no effect on your current or future health care at Shands Hospital at the University of Florida. If you have any questions about your rights as a research subject, you can phone the Institutional Review Board at 352-273-9600.

Completion and return of the survey implies that you have read the information in this form and consent to take part in the research. Please keep this form for your records or future reference.

Directions: Please answer each question as best as you can by checking the appropriate box or letter.

1. Did you complete a $4^{\text {th }}$ year advanced endoscopy fellowship?
a) Yes
b) No

2. When did you complete your $4^{\text {th }}$ year advanced endoscopy fellowship?
a) $<3$ years ago
b) $3-10$ years ago
c) $>10$ years ago
d) Not applicable

3. Your endoscopy practice is primarily based at:

a) Community Hospital/Practice

b) Academic Medical Center

4. Veterans Administration Hospital

Approximately how many ERCPs do you perform annually?
a) $25-75$
b) $75-150$
c) $>150$

5. Approximately how many ERCPs for malignant distal biliary obstruction do you perform annually?
a) Less than 5
b) $6-10$ 
c) $10-20$

d) $>20$

6. What are the most common reasons for biliary decompression (ERCP) in patients with malignant distal biliary obstruction in your practice? Please rank the choices from 1 to 6 , with 1 being the MOST common and 6 being the LEAST common. Please use each number only once.

\begin{tabular}{|c|c|c|c|c|c|c|}
\hline & 1 & 2 & 3 & 4 & 5 & 6 \\
\hline Surgeon prefers lower total bilirubin prior to surgery & $\square$ & $\square$ & $\square$ & $\square$ & $\square$ & $\square$ \\
\hline Surgery will be delayed more than 2 weeks & $\square$ & $\square$ & $\square$ & $\square$ & $\square$ & $\square$ \\
\hline Neoadjuvant therapy prior to surgery & $\square$ & $\square$ & $\square$ & $\square$ & $\square$ & $\square$ \\
\hline Patient symptoms (i.e. severe pruritus) & $\square$ & $\square$ & $\square$ & $\square$ & $\square$ & $\square$ \\
\hline Patient has ascending cholangitis (i.e. fever, jaundice, abdominal pain) & $\square$ & $\square$ & $\square$ & $\square$ & $\square$ & $\square$ \\
\hline Standard of care at your institution & $\square$ & $\square$ & $\square$ & $\square$ & $\square$ & $\square$ \\
\hline
\end{tabular}

7. How often do surgeons refer you patients with RESECTABLE malignant distal biliary obstruction for preoperative biliary drainage (ERCP) prior to surgery?
a) All of the time
b) Most of the time
c) Some of the time
d) Rarely
e) Never

8. What type of stent do you most commonly use for the management of a RESECTABLE malignant distal biliary obstruction?
a) Plastic
b) Partially covered metal stent
c) Fully covered metal stent
d) Uncovered metal stent

9. What type of stent do you most commonly use for the management of an UNRESECTABLE malignant distal biliary obstruction (palliative purposes)?
a) Plastic
b) Partially covered metal stent
c) Fully covered metal stent
d) Uncovered metal stent

10. Does the type of stent depend on whether the patient still has a gallbladder?
a) Yes
b) No

11. When placing a covered biliary stent, do you avoid covering the cystic duct take-off if the patient still has a gallbladder?
a) Yes
b) No

12. Do you commonly perform biliary sphincterotomy prior to stent insertion for the management of malignant distal biliary obstruction?
a) All of the time
b) Most of the time
c) Some of the time
d) Rarely
e) Never

13. Do you commonly perform biliary sphincterotomy prior to stent insertion if the biliary obstruction is from pancreatic cancer?
a) All of the time
b) Most of the time
c) Some of the time
d) Rarely
e) Never

14. For RESECTABLE malignant distal biliary obstruction: what is your approach if first attempt at ERCP fails?

a) Repeat ERCP by yourself or at another center

b) Refer for percutaneous transhepatic cholangiography 
c) Refer to surgery

d) EUS-guided biliary drainage by yourself or at another center

15. For UNRESECTABLE malignant distal biliary obstruction: what is your approach if first attempt at ERCP fails?

a) Repeat ERCP by yourself or at another center

b) Refer for percutaneous transhepatic cholangiography

c) Refer to surgery

d) EUS-guided biliary drainage by yourself or at another center 